\title{
Prevenção e terapêutica no tratamento de lesões por pressão: uma revisão integrativa
}

Prevention and therapeutic in the treatment of pressure injuries: an integrative review

Prevención y terapéutica en el tratamiento de las lesiones por presión: una revisión integrativa

Danielle Mota Bastos ${ }^{1 *}$, Joana D’arc Lyra Batista ${ }^{1}$, José Alex Alves dos Santos ${ }^{1}$, Marcela Lourene Correia Muniz ${ }^{1}$, Maria Luísa Corrêa Muniz ${ }^{1}$, Patricia Maria de Oliveira Andrade Araújo ${ }^{1}$, Ivanise Sales Rodrigues $^{1}$, Ana Carolina das Chagas Moura ${ }^{1}$, Kallyne Vitória Monteiro da Silva ${ }^{1}$, Thaiza de Lima Souto ${ }^{1}$.

\section{RESUMO}

Objetivo: Avaliar as terapêuticas utilizadas na prevenção e/ou tratamento de lesão por pressão, com a finalidade de analisar sua ocorrência, bem como, descrever medidas preventivas e tratamentos eficazes. Métodos: Trata-se de uma revisão integrativa da literatura realizada nas bases de dados: LILACS, MEDLINE, BVS, BDENF. Foram selecionados 16 artigos no período de 2010 a 2020 em português, inglês e espanhol que tratavam sobre prevenção e terapêutica de lesão por pressão. Resultados: Devido ao baixo custo e a melhor qualidade de vida do paciente as medidas preventivas: uso de escalas de risco, protocolos de tratamento e educação permanente devem ser adotadas. Todavia, o portador de lesão por pressão pode ter acesso a diferentes técnicas, curativos e coberturas. Tais procedimentos deverão ser realizados por profissionais devidamente treinados, considerando a avaliação clínica da lesão e a indicação do produto e/ou técnica a ser utilizada. Considerações finais: Apesar dos avanços tecnológicos são necessários maiores investimentos por partes dos gestores dos serviços de saúde e um maior comprometimento dos membros da equipe multiprofissional para utilizarem os recursos já conhecidos e eficazes para a prevenção e tratamento destas lesões a fim de preservar e/ou restabelecer a saúde do indivíduo que precisa de cuidados.

Palavras-chave: Terapêutica, Lesão por pressão, Enfermagem.

\begin{abstract}
Objective: Evaluate the therapies used in the prevention and/or treatment of pressure injuries, in order to analyze their occurrence, as well as describe preventive measures and effective treatments. Methods: This is an integrative literature review carried out in the following databases: LILACS, MEDLINE, BVS, BDENF. Sixteen articles were selected from 2010 to 2020 in Portuguese, English and Spanish dealing with pressure injury prevention and therapy. Results: Due to the low cost and better quality of life of the patient, preventive measures: use of risk scales, treatment protocols and continuing education should be adopted. However, patients with pressure injuries can have access to different techniques, dressings and dressings. Such procedures must be performed by properly trained professionals, considering the clinical evaluation of the lesion and the indication of the product and/or technique to be used. Final considerations: Despite technological advances, greater investments are needed by health service managers and greater commitment by members of the multidisciplinary team to use known and effective resources for the prevention and treatment of these injuries in order to preserve and/or restore the health of the individual in need of care.
\end{abstract}

Keywords: Therapeutics, Pressure ulcers, Nursing.

\section{RESUMEN}

Objetivo: Evaluar las terapias utilizadas en la prevención y / o tratamiento de las lesiones por presión, con el fin de analizar su ocurrencia, así como describir las medidas preventivas y tratamientos efectivos. Métodos: Se trata de una revisión integrativa de la literatura realizada en las siguientes bases de datos: LILACS, MEDLINE, BVS, BDENF. Se seleccionaron dieciséis artículos de 2010 a 2020 en portugués, inglés y español que tratan sobre la prevención y el tratamiento de las lesiones por presión. Resultados: Debido al bajo costo

${ }^{1}$ Instituto Federal de Educação Ciência e Tecnologia de Pernambuco, Abreu e Lima - PE.

*E-mail: danielle.mota@abreuelima.ifpe.edu.br

SUBMETIDO EM: 6/2021

ACEITO EM: 7/2021

PUBLICADO EM: 7/2021 
y la mejor calidad de vida del paciente, se deben adoptar medidas preventivas: uso de escalas de riesgo, protocolos de tratamiento y educación continua. Sin embargo, los pacientes con lesiones por presión pueden tener acceso a diferentes técnicas, apósitos y apósitos. Dichos procedimientos deben ser realizados por profesionales debidamente capacitados, considerando la evaluación clínica de la lesión y la indicación del producto y / o técnica a utilizar. Consideraciones finales: A pesar de los avances tecnológicos, se necesitan mayores inversiones por parte de los gerentes de servicios de salud y un mayor compromiso por parte de los miembros del equipo multidisciplinario para utilizar recursos conocidos y efectivos para la prevención y tratamiento de estas lesiones con el fin de preservar y / o restaurar la salud del individuo que necesita atención.

Palabras clave: Terapéutica, Lesión por presión, Enfermería.

\section{INTRODUÇÃO}

Lesão por Pressão (LPP) conforme estabelecido pelo National Pressure Ulcer Advisory Panel (2016) é o termo referente ao dano cutâneo, que a depender de sua gravidade pode acometer tecidos mais profundos, podendo haver a exposição da fáscia, músculo, tendão, ligamento, cartilagem e/ou osso. As lesões geralmente são situadas sobre proeminências ósseas, entretanto outros artefatos como Equipamentos de Proteção Individual e dispositivos médicos, podem ser os agentes causadores da lesão. Tais lesões podem ser classificadas em estágios de acordo com suas características, podendo ser mais superficiais (estágio I); perpassando pela exposição da derme (estágio II); seguindo pela perda total do tecido epitelial (estágio III); até níveis de degradação tecidual da fáscia muscular ao tecido ósseo (estágio IV).

A etiologia destas lesões está diretamente relacionada à qualidade da assistência prestada ao paciente, gerando um grande impacto em seu bem-estar, que pode ser evitado com métodos preventivos, além de diminuir o tempo de internação do paciente. Sendo assim, as LPP são consideradas um problema crônico de saúde pública, pois perpassa os mais diversos âmbitos assistenciais, principalmente em ambientes hospitalares o que compromete a segurança e a qualidade de vida do paciente. Por essa razão, através de estudos, houve grandes avanços no entendimento e, consequentemente, na terapêutica adotada nas diversas fases do processo de reparo tecidual (SANTOS CT, et al., 2013; SILVA ACO, et al., 2017).

Neste contexto, a equipe de Enfermagem tem grande importância na prevenção e tratamento das LPP, pois tem uma atuação de forma direta e contínua ao indivíduo doente. Ao mesmo tempo, sabe-se que estes profissionais em muitas instituições, públicas e privadas, trabalham com insuficiência de materiais e equipamentos para tal, assim como, vivenciam uma sobrecarga de trabalho dificultando assim, uma assistência segura e com qualidade (FREITAS VF, 2013, PACHÁ HHP, et al., 2018).

Atualmente é observado uma alta incidência de LPP, relacionada ao aumento da expectativa de vida da população, a internação por doenças infecciosas, parasitárias e neoplasias e a períodos de internação maiores que sete dias. Por isso, a revisão dos estudos com ênfase na temática se faz importante, ampliando o conhecimento e trazendo novas possibilidades acerca da prevenção e tratamento das LPP. Portanto à necessidade de obter mais conhecimento acerca da temática, visando contribuir para um atendimento integral, eficaz e acima de tudo humanizado nas instituições (PACHÁ HHP, et al., 2018).

Diante dos aspectos citados, este estudo tem como objetivo realizar uma revisão integrativa da literatura sobre as medidas de prevenção e/ou tratamento de LPP, reunindo as evidências científicas encontradas com a finalidade de analisar sua ocorrência, bem como, descrever medidas preventivas e tratamentos eficazes.

\section{MÉTODOS}

Trata-se de uma revisão integrativa da literatura, de natureza qualitativa, sendo utilizado um recorte temporal em publicações científicas do ano de 2010 a 2020 tendo em vista ter sido o período com expressivo número de estudos publicados sobre medidas preventivas e terapêuticas para LPP. A revisão Integrativa de literatura é considerada um método que conduz ao conhecimento de uma forma mais condensada e agregase na finalidade da obtenção de resultados significativos aos profissionais quanto a temática na prática da atenção em saúde (SOUZA MT, et al., 2010). 
Para atender o objetivo foram consultadas as publicações indexadas nas bases de dados eletrônicas Literatura Latino-Americana e do Caribe em Ciências da Saúde (LILACS), Medical Literature Analysis and Retrieval Sistem (MEDLINE) e Biblioteca Virtual de Saúde (BVS) e Base de dados em Enfermagem (BDENF). Utilizaram-se os seguintes descritores de acordo com os Descritores em Ciências da Saúde (DeCS): Terapêutica, Lesão por Pressão e Enfermagem.

O recurso utilizado na pesquisa foi a expressão do "título, resumo, assunto". Para o cruzamento dos termos foi utilizado o operador lógico booleano "and" com o objetivo de obter um maior número de artigos que respondessem à tal questão: Quais são os métodos de prevenção e de tratamento para as lesões por pressão?

Foram incluídos os estudos completos, com acesso livre, no idioma inglês, português e espanhol e excluídos os que possuíam alguma taxa de pagamento ou que estivesse incompleto. Procederam-se às etapas da revisão integrativa da literatura e após a coleta dos dados os artigos foram organizados e agrupados por semelhança no conteúdo e os resultados descritos. Os documentos encontrados através da busca foram contabilizados e expressos através de um fluxograma (Figura 1).

Figura 1 - Fluxograma da estratégia de busca da revisão integrativa da base de dados.

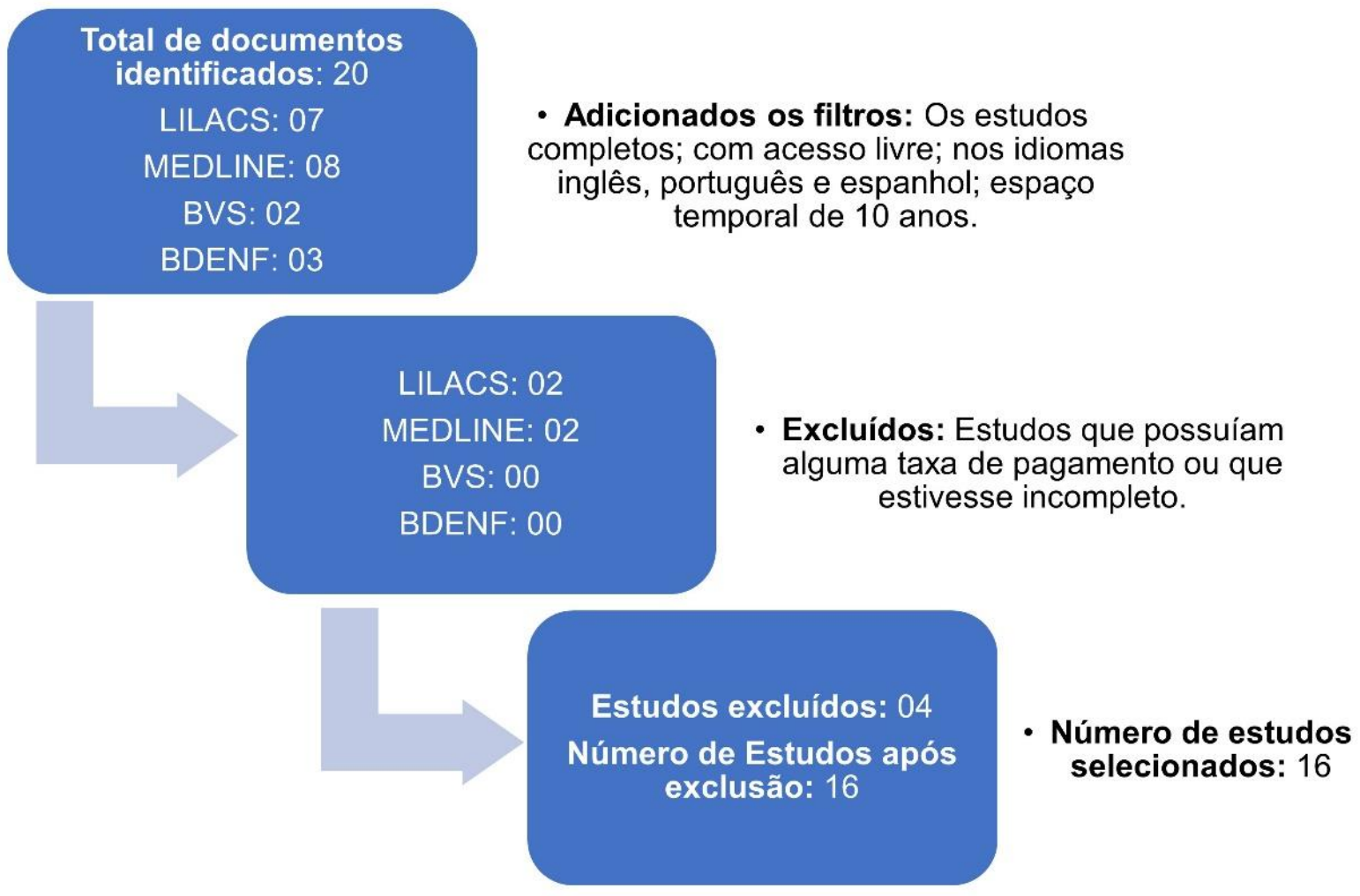

Fonte: Bastos DM, et al., 2021.

\section{RESULTADOS}

Do total de 16 artigos selecionados, oito foram realizados no Brasil, três na Espanha, um nos Estados Unidos, um na Finlândia, um na Alemanha, um no Reino Unido e um na China. Entre os estudos, nove foram publicados em periódicos do Brasil, três no Reino Unido, três na Espanha e um nos Estados Unidos. Em relação ao idioma obteve-se: seis em português, sete em inglês e três em espanhol. Ao todo, $37,5 \%$ dos estudos foram ensaios clínicos controlados, $25 \%$ de abordagem quantitativa, $12,5 \%$ de abordagem qualitativa, $12,5 \%$ foram estudo de caso e $12,5 \%$ foram estudos descritivos. O Quadro 1 demonstra estes dados descritos e suas variáveis. 
Quadro 1. Estudos selecionados para a revisão.

\begin{tabular}{|c|c|c|c|c|c|}
\hline Bases & Autores/Local/Ano & Temática/Considerações & Tipo de Estudo & $\begin{array}{l}\text { STROBE/Nível } \\
\text { de Evidência }\end{array}$ & Principais Resultados \\
\hline LILACS & $\begin{array}{c}\text { (SOUZA IMC, } \\
\text { et al., 2020, Brasil) }\end{array}$ & $\begin{array}{l}\text { Identificar os fatores facilitadores e } \\
\text { dificultadores para a prevenção e } \\
\text { tratamento da lesão por pressão (LP). }\end{array}$ & $\begin{array}{l}\text { Estudo } \\
\text { transversal } \\
\text { quantitativo }\end{array}$ & $\mathrm{B} ; \mathrm{VI}$ & $\begin{array}{l}\text { Quanto ao tratamento, pouco mais de } 60 \% \\
\text { referem que o paciente e a lesão são } \\
\text { avaliados por enfermeiros. }\end{array}$ \\
\hline MEDLINE & $\begin{array}{l}\text { (STOLT M, et al., } 2019, \\
\text { Finlândia) }\end{array}$ & $\begin{array}{c}\text { Examinar o estado do tratamento de UP } \\
\text { existente. }\end{array}$ & $\begin{array}{l}\text { Estudo } \\
\text { transversal } \\
\text { qualitativo }\end{array}$ & $A ; V I$ & $\begin{array}{l}\text { O tratamento da UP é inconsistente e } \\
\text { frequentemente realizado com métodos e } \\
\text { produtos variados. }\end{array}$ \\
\hline BDENF & $\begin{array}{l}\text { (ROCHA DM, et al., } \\
\text { 2018, Brasil) }\end{array}$ & $\begin{array}{l}\text { Avaliar o custo da terapia tópica de } \\
\text { pacientes com lesão por pressão. }\end{array}$ & $\begin{array}{l}\text { Estudo } \\
\text { quantitativo }\end{array}$ & $\mathrm{B} ; \mathrm{VI}$ & $\begin{array}{l}\text { Mostrou-se que a lesão por pressão } \\
\text { apresentou alto custo }\end{array}$ \\
\hline BDENF & $\begin{array}{l}\text { (CARVALHO MRF, et } \\
\text { al., 2017, Brasil) }\end{array}$ & $\begin{array}{l}\text { Construir algoritmo para tratamento da } \\
\text { lesão por pressão (LPP) }\end{array}$ & $\begin{array}{c}\text { Estudo } \\
\text { Quantitativo }\end{array}$ & $\mathrm{B} ; \mathrm{VI}$ & $\begin{array}{l}92,1 \% \text { dos participantes opinaram } \\
\text { favoravelmente }\end{array}$ \\
\hline LILACS & $\begin{array}{l}\text { (SOUZA ACS, et al., } \\
\text { 2017, Brasil) }\end{array}$ & $\begin{array}{c}\text { Avaliar a eficácia da estimulação elétrica } \\
\text { de alta voltagem. }\end{array}$ & Estudo de Caso & $B ;$ IV & $\begin{array}{l}\text { Os resultados mostraram uma porcentagem } \\
\text { de } 17 \% \text { de redução. }\end{array}$ \\
\hline MEDLINE & $\begin{array}{l}\text { (OMAR M, et al., 2016, } \\
\text { Alemanha) }\end{array}$ & Comparar o impacto da instilação salina & $\begin{array}{l}\text { Ensaio Clínico } \\
\text { Controlado }\end{array}$ & $\mathrm{B}, \mathrm{II}$ & Verificou-se menor tempo de internação. \\
\hline MEDLINE & $\begin{array}{l}\text { (GUIHAN M, et al., } \\
\text { 2016, Estados Unidos) }\end{array}$ & $\begin{array}{l}\text { Identificar características associadas à } \\
\text { cicatrização de úlcera por pressão }\end{array}$ & $\begin{array}{l}\text { Ensaio Clínico } \\
\text { Controlado }\end{array}$ & C, II & $\begin{array}{l}\text { Os modelos de regressão de Poisson, só o } \\
\text { estágio da úlcera prévia } 50 \% \text { e } 100 \% \text { de } \\
\text { cicatrização nas semanas } 4 \text { e } 12 \text {. }\end{array}$ \\
\hline LILACS & $\begin{array}{l}\text { (ANDRADE CCD, et } \\
\text { al., 2016, Brasil) }\end{array}$ & $\begin{array}{l}\text { Realizar uma avaliação } \\
\text { do custo do tratamento tópico }\end{array}$ & $\begin{array}{l}\text { Estudo } \\
\text { Quantitativo }\end{array}$ & $\mathrm{B}, \mathrm{IV}$ & $\begin{array}{l}\text { Os custos do procedimento variaram entre } \\
\text { BRL } 16,41 \text { e BRL } 260,18 \text {. }\end{array}$ \\
\hline LILACS & $\begin{array}{l}\text { (PALAGI S, } \\
\text { 2015, Brasil) }\end{array}$ & Descrever o processo de cicatrização & Estudo de Caso & $\mathrm{B}, \mathrm{II}$ & $\begin{array}{l}\text { Redução da lesão de } 7 \mathrm{~cm} \text { para } 1,5 \mathrm{~cm} \text { de } \\
\text { comprimento e de } 6 \mathrm{~cm} \text { para } 1,1 \mathrm{~cm} \text { de largura }\end{array}$ \\
\hline
\end{tabular}




\begin{tabular}{|c|c|c|c|c|c|}
\hline Bases & Autores/Local/Ano & Temática/Considerações & Tipo de Estudo & $\begin{array}{l}\text { STROBE/Nível } \\
\text { de Evidência }\end{array}$ & Principais Resultados \\
\hline MEDLINE & $\begin{array}{l}\text { (DUMVILLE JC, 2015, } \\
\text { Reino Unido) }\end{array}$ & Avaliar os efeitos dos curativos de alginato & $\begin{array}{l}\text { Ensaio clínico } \\
\text { controlado. }\end{array}$ & $A, V$ & $\begin{array}{c}\text { Não há evidências de efeitos diferenciais no } \\
\text { processo de cicatrização }\end{array}$ \\
\hline MEDLINE & $\begin{array}{l}\text { (WEIZHONG L, et al., } \\
\text { 2014, China) }\end{array}$ & $\begin{array}{c}\text { Investigar o tratamento da úlcera de } \\
\text { decúbito sacral através de retalho muscular } \\
\text { e irrigação fechada. }\end{array}$ & $\begin{array}{l}\text { Estudo } \\
\text { descritivo. }\end{array}$ & $\mathrm{B}, \mathrm{VI}$ & $\begin{array}{l}\text { Útil no tratamento de úlceras de decúbito } \\
\text { sacral. }\end{array}$ \\
\hline BVS & $\begin{array}{l}\text { (DÍAZ-VALENZUELA } \\
\text { AD, et al., } \\
\text { 2014, Espanha) }\end{array}$ & $\begin{array}{c}\text { Avaliar a eficácia da aplicação tópica de } \\
\text { azeite de oliva extravirgem na prevenção } \\
\text { de UPP. }\end{array}$ & $\begin{array}{l}\text { Ensaio clínico } \\
\text { controlado. }\end{array}$ & $A, I I$ & $\begin{array}{l}\text { Os resultados apontaram a primeira } \\
\text { evidência sobre a eficácia }\end{array}$ \\
\hline LILACS & $\begin{array}{l}\text { (ARRUDA FCF, 2013, } \\
\text { Brasil) }\end{array}$ & $\begin{array}{l}\text { Relatar a experiência com o uso de } \\
\text { retalhos da região glútea }\end{array}$ & $\begin{array}{l}\text { Estudo } \\
\text { qualitativo. }\end{array}$ & $\mathrm{B}, \mathrm{VI}$ & $\begin{array}{l}\text { Uma ótima opção, beneficiando o paciente } \\
\text { em sua recuperação e reabilitação. }\end{array}$ \\
\hline BDENF & $\begin{array}{c}\text { (POTT FS, et al., 2013, } \\
\text { Brasil) }\end{array}$ & $\begin{array}{l}\text { Descrever o desenvolvimento de um } \\
\text { algoritmo para a prevenção e tratamento } \\
\text { de úlceras por pressão. }\end{array}$ & $\begin{array}{l}\text { Estudo } \\
\text { Descritivo }\end{array}$ & $\mathrm{A}, \mathrm{VI}$ & $\begin{array}{c}\text { Sequência descrita em três etapas: Avaliação } \\
\text { da Enfermagem, Ações de Cuidado e } \\
\text { Proposta Terapêutica. }\end{array}$ \\
\hline BVS & $\begin{array}{l}\text { (ARÉVALO VELASCO } \\
\text { JM, et al., 2012, } \\
\text { Espanha) }\end{array}$ & $\begin{array}{l}\text { Realizar a detecção transcutânea de } \\
\text { oxigênio }\left(\mathrm{TcpO}_{2}\right) \text { em } 11 \text { pacientes }\end{array}$ & $\begin{array}{l}\text { Ensaio Clínico } \\
\text { Controlado }\end{array}$ & $B, \|$ & $\begin{array}{l}\text { Na posição pré-operatória } 1 \text {, os valores } \\
\text { obtidos foram } 27,28 \pm 13,83 \mathrm{mmHg} \text {, enquanto } \\
\text { no } \mathrm{P} 1 \text { pós-operatório eles eram } 34,96 \pm 19,06 \\
(\mathrm{p}<0,05) .\end{array}$ \\
\hline MEDLINE & $\begin{array}{l}\text { (CANDELA-ZAMORA } \\
\text { MD, et al., 2010 } \\
\text { Espanha) }\end{array}$ & $\begin{array}{c}\text { Avaliar se existe uma equivalência entre } \\
\text { dois tratamentos baseados em ácidos } \\
\text { graxos hiperoxigenados (AGHO), AGHO e } \\
\text { Mepentol }{ }^{\circledR} \text { (fitoterápico) }\end{array}$ & $\begin{array}{l}\text { Ensaio Clínico } \\
\text { Controlado }\end{array}$ & $\mathrm{B}, \mathrm{II}$ & $\begin{array}{l}148 \text { úlceras (72 controles e } 76 \text { intervenções) } \\
\text { completaram o estudo. } 40 \text { úlceras }(55,6 \%) \\
\text { cicatrizaram no grupo controle e } 53(69,7 \%) \\
\text { no grupo intervenção } p=0,074 \text {. }\end{array}$ \\
\hline
\end{tabular}

Fonte: Bastos DM, et al., 2021; dados extraídos das bases de dados PubMed, LILACS e SciELO. 


\section{DISCUSSÃO}

Sendo uma das principais causas de morbimortalidade, incapacidades, dependência de cuidados e hospitalização, o surgimento de LPP, como já se pode ter notado, é multicausal, podendo ser evitável, até porque está diretamente influenciado por fatores intrínsecos e extrínsecos e sua classificação indica o grau de comprometimento tecidual, variando nos estágios 1, 2, 3, 4 e duas condições adicionais: a lesão não classificável e a tissular profunda (CALIRI MHL, et al., 2016).

Clinicamente, pôde ser visto no presente estudo de revisão, que estas lesões são predominantemente classificadas como feridas crônicas (estágio $4-72,2 \%)$, com maiores frequências nas regiões sacral (48,6\%), trocânter (19,2\%) e calcâneo (19,2\%) (FREITAS VF, et al., 2013). A maior prevalência na região sacral devese a alta pressão local, sendo um local de difícil descompressão e maior suscetibilidade de distribuição desigual do peso corporal associadas à fatores de risco, deficiências no cuidado, ausência de recursos materiais para realização dos curativos e, principalmente, uma avaliação clínica tardia, o que pode favorecer ainda mais a necrose tecidual (SILVA ACO et al., 2017).

No entanto, também foi relatado que tratar uma LPP torna-se mais oneroso que aplicar medidas preventivas, já que estas últimas evitam a instalação do processo lesivo e impedem o aparecimento de complicações como processos infecciosos (ANDRADE CCD, et al., 2016; ROCHA DM, et al., 2018). Por isso, estas lesões podem ser consideradas um problema de saúde pública já que segundo a National Pressure Ulcer Advisory Panel (NPUAP, 2016), a incidência em hospitais dos Estados Unidos é de 3 a 14\%, já nas instituições de longa permanência é de 15 a 25\%. No cenário mundial essa incidência varia de 1,9 a 7\% em ambientes hospitalares (GARDINER JC, et al., 2016).

Predominantemente, as LPP são tratadas e avaliadas por uma equipe multiprofissional, porém destacando a equipe de enfermagem, já que pouco mais de 60\% das LPP são avaliadas por enfermeiros (SOUZA MC, et al., 2020). Estes por sua vez, com sua visão holística, participam de todos os processos do cuidar, desde as medidas profiláticas, gestão de recursos, terapêutica e a reabilitação (PACHÁ HHP, et al., 2018).

Outro ponto importante está relacionado aos tratamentos aplicados às LPP, encontrados nesta revisão, em sua maioria ensaios clínicos, no qual se notou que apesar dos bons resultados obtidos com as inovações tecnológicas realizadas, algumas terapêuticas mais tradicionais, como uso de ácidos graxos e cirurgias reparadoras, demonstraram não serem inertes ao processo de reparo tecidual. Por essas razões os tratamentos das LPP são considerados inconsistentes e frequentemente realizados com métodos e produtos variados (CANDELA-ZAMORA MD, et al., 2010; ARÉVALO JM, et al., 2012; ARRUDA FCF, et al., 2013; DÍAZVALENZUELA AD, et al., 2014; SOUZA ACS, et al., 2017; STOLT M, et al., 2019).

\section{Medidas de prevenção}

Considerando os baixos custos com medidas preventivas das LPP, é essencial que a equipe de saúde elabore instrumentos educativos, como por exemplo, protocolos, manuais, cartilhas, folhetos e algoritmos (DUTRA RA, et al., 2015; ANDRADE CCD, et al., 2016; LIMA AFC, et al., 2016; CARVALHO MRF, et al., 2017).

A educação permanente/continuada do profissional é uma das medidas mais seguras e viáveis na redução dos riscos de um paciente desenvolver LPP. Para tanto, na área de saúde, se deve trabalhar com ferramentas que busquem refletir a mudança no espaço de trabalho como uma estratégia de transformação efetiva na prática profissional. Entretanto, é notável que nas instituições de saúde há um baixo investimento na qualificação do sujeito e na aquisição/desenvolvimento de novas tecnologias para prevenção de tais lesões (SOUZA MC, et al., 2020).

Neste contexto, o uso de diretrizes e a implantação de protocolos preventivos são considerados como a melhor estratégia para o fortalecimento das práticas assistenciais, ampliando as práticas preventivas e reduzindo, consequentemente, a ocorrência de complicações da LPP (LIMA AFC, et al., 2016; CARVALHO MRF, et al., 2017).

A adoção de condutas preventivas, através de instrumentos como escalas de risco e protocolos/diretrizes para desenvolvimento de LPP, já demonstraram que auxiliam os enfermeiros a avaliar o risco, formular o 
diagnóstico e determinar o plano de cuidados (POTT FS et al., 2013). Neste contexto, na literatura há em torno de 40 escalas de avaliação de risco para LPP. Todavia, as mais conhecidas são as de Norton, Waterlow e Braden (ARAÚJO TM, et al. 2011).

Contudo, estudos que confrontam o uso destas escalas preventivas em quase sua totalidade foram desenvolvidos na Europa (Grã-Bretanha, Alemanha e Holanda) e Ásia (China), sendo encontrado apenas um estudo brasileiro que compara os resultados das escalas de Norton, Braden e Waterlow (ARAÚJO TM, et al., 2011). Por estes estudos, foi possível inferir que os usos dessas ferramentas possibilitam uma melhor conduta profilática/terapêutica na avaliação clínica da equipe multiprofissional, em especial a de enfermagem.

Desta forma, dentre os artigos selecionados, apenas dois estudos apresentaram propostas de aplicação profilática dos algoritmos, sendo ambos desenvolvidos em hospitais universitários do Brasil. O primeiro estudo utilizou os pressupostos da escala de Braden para a construção do seu algoritmo, já o segundo estudo realizou um levantamento bibliográfico em bases científicas e construiu seu algoritmo baseado em quatro etapas: avaliação da lesão por pressão; limpeza da lesão por pressão; classificação da lesão por pressão; e proposta do agente terapêutico (POTT FS, et al., 2013; CARVALHO MRF, et al., 2017).

Com esses estudos pode-se inferir que independentemente da medida profilática adotada algumas ações são essenciais para a manutenção da integridade da pele, como a inspeção, manejo da umidade e hidratação e higiene; além disso, medidas de redução de pontos de pressão, como o uso do relógio de decúbito e colchões específicos (casca de ovo e/ou pneumáticos). Entretanto, tais medidas requerem ações educativas e cooperativas entre a equipe multiprofissional, a família e o próprio paciente.

\section{Medidas Terapêuticas}

No presente estudo, foi possível identificar diversas terapêuticas empregadas com intuito de cicatrizar uma LPP. A saber, foram citados uso de tecnologias como: Estimulação Elétrica de Alta Voltagem (EEAV), Laserterapia, NPWTi (Negative Pressure Wound Therapy With Instillation) e $\mathrm{TcpO}_{2}$ (Transcutaneous oxygen pressure). Além disso, o uso de coberturas: Ácidos Graxos Essenciais (AGE), Alginato, Hidrocoloide, Hidrogel, Carvão Ativado, Cobertura não aderente, Malha de Prata e Espuma de Poliuretano. E terapia nãoconvencional, a Fitoterapia (CANDELA-ZAMORA MD, et al., 2010; ARÉVALO JM, et al., 2012; ARRUDA FCF, et al., 2013; DÍAZ-VALENZUELA AD, et al., 2014; SOUZA ACS, et al., 2017; STOLT M, et al., 2019).

O uso de EEAV é uma forma de administração de estímulos elétricos para auxiliar o processo de reparo tecidual, visto que, no processo de injúria tecidual as correntes elétricas endógenas são alteradas gerando os chamados "sinais de erro". Para tanto, a EEAV estimula a autorreparação elétrica que disparam o processo de reparação tecidual (MAíSA SG, et al., 2013).

Sendo considerada pela NPUAP uma das formas de tratamento complementares para LPP, a laserterapia age através de uma radiação de luz amplificada de baixa frequência promovendo efeitos bioquímicos, bioelétricos e bioenergéticos (DISSEMOND J, 2010). Para a literatura, assim como a EEAV, a laserterapia é considerada segura, eficaz e com potencial cicatrizante, analgésico e anti-inflamatório para as LPP, aumentando o tecido de granulação e reduzindo a área da ferida (ANDRADE FSSD, et al., 2014; PALAGI S, et al., 2015).

Já a Terapia por Pressão Negativa (NPWT), têm a sua aplicação de vácuo na superfície de uma ferida através de um corte de curativo de espuma para ajustar a ferida. Isso remove o exsudato da ferida, reduz o acúmulo de mediadores inflamatórios e aumenta o fluxo de nutrientes para a ferida, promovendo a cura (GATHEN M, et al., 2016).

Visto que as medidas preventivas são menos onerosas, foi verificado que os custos com a terapêutica empregada no tratamento das LPP apresentam uma relação diretamente proporcional ao estágio da lesão. Apresentando maiores custos quando há a presença de tecido desvitalizado, exsudato intenso e odor fétido, já que requerem trocas de curativos com maior frequência (ROCHA et al., 2018). Além disso, também há um custo secundário na formação técnico-científica dos profissionais para o melhor manuseio do material adquirido (CARVALHO MRF, et al., 2017).

Para tanto, na tentativa de aumentar a eficiência terapêutica dos tratamentos adotados, a técnica da $\mathrm{TcpO}_{2}$ é citada na literatura como um guia-terapêutico, sendo testada em pacientes paraplégicos portadores de LPP 
de estágios 3-4 que iriam ser submetidos a um desbridamento cirúrgico. Com o uso da técnica foi possível inferir que nas lesões pré-operatórias os níveis transcutâneos de oxigênio eram inferiores às lesões pósoperatórias, demonstrando a eficiência da técnica cirúrgica (ARÉVALO JM, et al., 2012).

Dentre os produtos utilizados na prevenção e/ou tratamento de LPP estão os AGE, este por sua vez mantém a pele hidratada, prevenindo a abertura de lesões. $E$ em lesões abertas, aceleram o processo de angiogênese e fibroplasia, podendo ser utilizado em LPP com ou sem infecções. Contudo, na literatura é possível encontrar estudos que associam ácidos graxos hiperoxigenados a fitoterápico e/ou comparado ao uso de azeite de oliva. Em ambos os estudos, tanto as terapêuticas isoladas quanto associadas demonstraram-se relevantes perante as LPP (CANDELA-ZAMORA MD, et al., 2010; DÍAZ-VALENZUELA AD, et al., 2014). Outros produtos bastante utilizados em ambientes hospitalares e não hospitalares são os agentes desbridantes, estes promovem uma ação enzimática (colagenase e papaína) ou autolítica (hidrogel, hidrocolóide e alginato de cálcio) (BUZZI M, et al., 2016).

Neste contexto, o alginato é uma opção para o tratamento da LPP, pois é altamente absorvente e, portanto, pode absorver o fluido (exsudato) produzido por algumas úlceras. Curativos de alginato apresentam-se na forma de alginato de cálcio ou alginato de cálcio e sódio, podendo ser combinados com colágeno (CONSTANTE SAR e OLIVEIRA VC, 2018). O alginato forma um gel quando em contato com a superfície da ferida, que pode ser retirado na remoção do curativo ou enxaguado com solução salina estéril (DUMVILLE JC, et al., 2015).

De uma forma geral dentre as coberturas mais utilizados, suas vantagens e desvantagens o uso de filme transparente mostrou-se uma opção mais econômica diante das placas de hidrocoloide em pacientes internados em Unidade de Terapia Intensiva (UTI), já que nesta última cobertura a periodicidade de trocas é menor, gerando um maior custo na terapêutica. Por essa razão, estudos como este, realizam comparações de eficácia, perante a profilaxia e ao tratamento das LPP, e principalmente relacionados aos ônus gerados pela escolha terapêutica (INOUE KC e MATSUDA LM, 2016; FULBROOK P, et al., 2019).

É mais vantajoso, por exemplo, o hospital investir em coberturas de silicone multicamadas, pois reduz os custos anuais em $60 \%$. Um método de prevenção bastante utilizado e eficaz é a mobilização corporal, que leva conforto ao paciente e é de suma importância para prevenção de LPP. A UTI tem maiores gastos diários, pois os pacientes se encontram em estados mais críticos e ficam limitados (ARAÚJO M, et al., 2019).

Por outro lado, o investimento em coberturas como a espuma de poliuretano apresentou excelentes resultados quanto aos aspectos: conservação da umidade no leito da lesão (impermeabilidade da cobertura), redução do exsudato, desbridamento autolítico, oxigenação (angiogênese) e antiséptico local (antimicrobiano). Percebe-se que cada produto/cobertura utilizada tem suas indicações, mas também possuem suas limitações, principalmente, quando se faz uma avaliação prévia da lesão e há a indicação de um curativo ideal. A exemplo, ao comparar o uso da sulfadiazina de prata ao da espuma de poliuretano em lesões exsudativas viu-se que o processo cicatricial ocorreu em menor tempo quando se utilizou a espuma de poliuretano, já que a sulfadiazina mantem a umidade enquanto a espuma reduz o exsudato, retraindo as bordas da ferida (FRANCO VQ, 2018).

Como se pode notar, no campo terapêutico as feridas podem ser tratadas com os mais diversos produtos. Entretanto, o acesso a esses insumos nem sempre é possível, e a população recorre para as fontes alternativas, como os produtos naturais de origem animal e/ou vegetal. Especificamente no território brasileiro esta prática se expandiu devido aos efeitos colaterais de fármacos sintéticos, fácil acesso a fontes naturais e baixo custo do tratamento (KIM DJ, et al., 2015). Foi observado que algumas abordagens terapêuticas são empregadas com o intuito de auxiliar na cicatrização, como por exemplo o uso de plantas medicinais no campo da fitoterapia, a aplicação de terapia tópica, levando em consideração a qualidade de vida e bem-estar do cliente (SOUZA ACS, et al., 2017).

Ressalta-se ainda, que a biocompatibilidade da cobertura e a hidratação da pele são fatores considerados ideais para a proteção da ferida e, consequentemente, favorecem o processo de reparo tecidual. Tratandose das LPP, as condições do leito da lesão é que vai determinar qual melhor alternativa para prevenir ou mesmo tratar essas feridas. Atualmente, há uma diversidade de produtos no mercado que necessitam ser selecionados de acordo com o julgamento clínico do profissional a fim de promover uma assistência de qualidade, existindo a necessidade de individualizar cada caso, observar suas peculiaridades para eleger os melhores cuidados para prevenção e tratamento dessas lesões (CORREIA ASB e SANTOS ICB, 2019). 
Desta forma, o cuidado holístico do paciente deve ser o foco. A equipe de enfermagem em ambientes de cuidados de longa duração e unidades hospitalares pode se beneficiar de uma educação em serviço aprofundada com foco no tratamento de LPP. Entretanto, ainda há a necessidade de mais pesquisas sobre a competência da equipe multiprofissional no tratamento da LPP (STOLT M, 2019).

\section{CONSIDERAÇÕES FINAIS}

Os achados desta revisão demonstraram que diante dos custos e impacto na qualidade de vida do paciente a melhor terapêutica diante da LPP é a prevenção. Apesar da equipe multiprofissional, em especial a equipe de enfermagem, demonstrar preocupação com a qualidade da assistência prestada, se faz necessário um maior comprometimento ao utilizarem os recursos já conhecidos e eficazes. Conclui-se que a gestão dos serviços, que as vezes apresenta uma visão restrita do cuidar, deve investir em novas tecnologias para profilaxia/terapêutica da LPP, em protocolos preventivos, na elaboração de instrumentos educativos e na qualificação profissional. São necessários maiores estudos sobre esta temática para melhor relacionar a teoria e a prática profissional aos cuidados profiláticos e terapêuticos.

\section{REFERÊNCIAS}

1. ANDRADE CCD, et al. Costs of topical treatment of pressure ulcer patients. Rev Esc Enferm USP. 2016; 50(2): 292298.

2. ANDRADE FSSD, et al. Effects of low-level laser therapy on wound healing. Rev Col Bras Cir. 2014; 41(2): $129-33$.

3. ARAUJO M, et al. Análise de custo da prevenção e do tratamento de lesão por pressão: revisão sistemática. Revista Enfermagem Atual In Derme. 2019; 89(27): 1-12.

4. ARAÚJO TM. Comparação de escalas de avaliação de risco para úlcera por pressão em pacientes em estado crítico. Acta Paul Enferm. 2011; 24(5): 695-700.

5. ARÉVALO VELASCO JM, et al. Eficacia de la detección percutánea de oxígeno como guía terapéutico-quirúrgica en lesionados medulares con úlceras por presión crónicas: estudio preliminar. Cir. plást. iberolatinoam. 2012; 38(3): 265271.

6. ARRUDA FCF. Opções de retalhos da região glútea no tratamento da úlcera de pressão nas regiões isquiática e sacral. Rev. bras. cir. plást. 2013; 28(3): 476-482.

7. BUZZI M, et al. Cicatrização de úlceras por pressão com extrato Plenusdermax® de Calendula officinalis L. Rev Bras Enferm, 2016; 69(2): 250-257.

8. CALIRI MHL, et al. Classificação das Lesões por pressão - Consenso NPUAP 2016 - Adaptada Culturalmente para o Brasil. São Paulo: SOBEST/SOBENDE; 2016.

9. CANDELA-ZAMORA MD, et al. Estudio comparativo de efectividad de dos ácidos grasos hiperoxigenados en el tratamiento de úlceras de grado I em pacientes geriátricos hospitalizados Enferm Clin. 2010; 20(1): 10-16.

10. CARVALHO MRF, et al. Construção e validação de algoritmo para tratamento da lesão por pressão. Rev enferm UFPE on line. 2017; 11(10): 4171-83.

11. CONSTANTE, SAR, OLIVEIRA, VC. Lesão por pressão: uma revisão de literatura. Rev. Psicol Saúde e Debate. 2018; 4(2): 95-114.

12. CORREIA ASB, SANTOS IBC. Lesão por Pressão: Medidas Terapêuticas Utilizadas por Profissionais de Enfermagem. Revista Brasileira da Ciência da Saúde. 2019; 23(1): 33-42.

13. DÍAZ-VALENZUELA A, et al. Efficacy for preventing pressure ulcers of the virgin extra olive oil versus hyperoxygenated fatty acids: intermediate results from a noninferiority trial. Gerokomos. 2014; 25(2): 74-80.

14. DISSEMOND J. Physical treatment modalities for chronic leg ulcers. Hautarzt. 2010; 61 (5): 387-96.

15. DUMVILLE JC. Alginate dressings for treating pressure ulcers. São Paulo Medical Journal. 2015;133(5): 455.

16. DUTRA RA, et al. Using transparent polyurethane filmand hydrocolloid dressings to prevent pressure ulcers. J Wound Care. 2015; 24(6): 268-75.

17. FULBROOK $P$, et al. Effectiveness of prophylactic sacral protective dressings to prevent pressure injury: A systematic review and meta-analysis. Int J Nurs Stud. 2019; 89(19): 1-36.

18. FRANCO VQ. Tecnologia da espuma de poliuretano com prata iônica e sulfadiazina de prata: aplicabilidade em feridas cirúrgicas infectadas. J. res.: fundam. care. 2018; 10(2): 441-449.

19. FREITAS VF. A importância dos cuidados do técnico em enfermagem na prevenção de lesão por pressão em pacientes hospitalizados. Biblioteca Virtual de Saúde - Coleciona SUS. ID: biblio-942971. 2013; (0)23.

20. GARDINER JC, et al. Incidence of hospital-acquired pressure ulcers -a population-based cohort study. Int Wound J. 2016; 13(5): 809-20.

21. GATHEN M, et al. [Negative Pressure Wound Therapy with Instillation in the Treatment of Critical Wounds]. [Article in German] Z Orthop Unfall 2016; 154(2): 122-127. 
22. GUIHAN M, et al. Difficulty in Identifying Factors Responsible for Pressure Ulcer 15. Healing in Veterans With Spinal Cord Injury. Arch Phys Med Rehabil. 2016; 97(12): 2085-2094.

23. INOUE KC, MATSUDA LM. Cost of dressings for prevention of sacral pressure ulcers. Rev Bras Enferm. 2016; 69(4): 598-602.

24. LIMA AFC, et al. Direct cost of dressing for pressure ulcers in hospitalized patients. Rev Bras Enferm. 2016; 69(2): 269-75.

25. MAÍSA SG, et al. Estimulação elétrica de alta voltagem incrementa a cicatrização de lesões cutâneas crônicas: análise de seis casos. Fisioter Pesqui 2013; 20(3): 286-92.

26. NATIONAL PRESSURE ULCER ADVISORY PANEL (NPUAP). Pressure Ulcer Stages Revised. Washington, 2016. Disponível em: http://www.npuap.org/about-us/

27. OMAR M, et al. A comparative study of negative pressure wound therapy with and without instillation of saline on wound healing. J Wound Care. 2016; 25(8): 475-478.

28. PACHÁ HHP, et al. Lesão por Pressão em Unidade de Terapia Intensiva: estudo de caso-controle. Rev Bras Enferm. 2018; 71(6): 3027-34.

29. PALAGI S, et al. Laserterapia em úlcera por pressão: avaliação pelas Pressure Ulcer Scale for Healing e Nursing Outcomes Classification. Rev Esc Enferm USP · 2015; 49(5):826-833.

30. KIM DJ, et al. Cutaneous wound healing in aging small mammals: a systematic review. Wound Repar. Regen. 2015; 33(3): 318-339.

31. POTT FS, et al. Algoritmo de prevenção e tratamento de úlcera por pressão. Cogitare Enferm. 2013. 18(2): 238-44

32. ROCHA DM, et al. Custo da terapia tópica em pacientes com lesão por pressão. Revista Enfermargem. 2018; 12(10): 2555-63.

33. SANTOS CT, et al. Indicador de Qualidade assistencial de úlcera por pressão: análise de prontuário e de notificação de incidente. Rev Gaúcha Enferm.2013; 34(1): 111-8.

34. SILVA ACO, et al. As principais coberturas utilizadas pelo enfermeiro. Revista UNINGÁ. 2017; 53(2): 117-123.

35. SOUZA ACS, et al. Efeito da estimulação elétrica de alta voltagem para o tratamento de úlceras por pressão: um estudo experimental de caso único. Fisioter. Bras. 2017; 18(6); 667-I:685.

36. SOUZA MC, et al. Cultura organizacional: prevenção, tratamento e gerenciamento de risco da lesão por pressão. Rev Bras Enferm. 2020; 73(3).

37. SOUZA MT, et al. Revisão integrativa: o que é e como fazer. Einstein. 2010; 8(1): 102-6.

38. STOLT M, et al. Local treatment of pressure ulcers in long-term care: a correlational cross-sectional study. J Wound Care. 2019; 28(6): 409-415.

39. WEIZHONG L, et al. The combination application of space filling and closed irrigation suction in reconstruction of sacral decubitus ulcer. Int Surg. 2014; 99(5): 623-627. 